\title{
Experiments on molecular hydrogen formation on cold ISM dust
}

\author{
Lisseth Gavilan, Jean Louis Lemaire, Gianfranco Vidali $\dagger$ \\ LERMA/LAMAp, UMR 8112 du CNRS, de l'Observatoire de Paris et de l'Université de Cergy \\ Pontoise, 5 mail Gay Lussac, 95000 Cergy Pontoise, France \\ email: lisseth.gavilan@obspm.fr
}

\begin{abstract}
We investigate the formation of molecular hydrogen on ISM dust grains at $\mathrm{T}<10$ $\mathrm{K}$. We use laboratory methods to detect newly formed $\mathrm{D}_{2}$ that is rovibrationally excited at $\mathrm{v}$ " = $4, \mathrm{~J} "=2$. We discover that recombination is catalyzed by pre-existing molecules on the dust surface and present this an important process in the coldest regions of the ISM.
\end{abstract}

Keywords. astrochemistry — ISM: atoms — ISM: molecules — dust, extinction

Introduction: $\mathrm{H}_{2}$ formation on dust grains is a complex process, initiating the chemistry of interstellar clouds. Since the late 90s, experiments on $\mathrm{H}_{2}$ formation (e.g. the Vidali group) have complemented theoretical models. Our recent work examines the assumption of Langmuir kinetics on molecule-covered dust, which suggests that molecular hydrogen formation is halted as molecules progressively adsorb on a cold dust surface.

Methods: We use FORMOLISM, a ultra-high vacuum setup located at the Université de Cergy Pontoise. We detect rovibrationally excited newly-formed $\mathrm{D}_{2}$ molecules via $\operatorname{REMPI}(2+1)$ photons from a UV laser and confirm that recombination is promptly enhanced on pre-covered dust surfaces (up to $57 \pm 10 \%$ at $5.5 \mathrm{~K}$ ). We interpret this as a result of enhanced atomic diffusion on the surface, both thermal and quantum mechanical (fig. 1), which facilitates atomic recombination. This is due to the preferential occupation of the deeper adsorption sites by the pre-existing molecules. These experiments are done at astrophysically relevant thermal conditions, such as $\mathrm{T}_{\text {dust }}=5.5 \mathrm{~K}$ and $\mathrm{T}_{\text {gas }}=90$ $\mathrm{K}$ and on a synthesized silicate analogue. The dust surface is cooled by a closed-cycle helium cryostat and the atomic beam is produced by a microwave discharge.

Astrophysical implications: The heat release in the exothermic formation of $\mathrm{H}_{2}$ may act as a non-thermal desorption mechanism towards the center of molecular cores. This will have a direct effect on the location of the $\mathrm{H} / \mathrm{H}_{2}$ transition which is affected by the exponentially increasing formation rate as dust cools down in dense regions. In conclusion, we show that dust remains an active catalyst even in the coldest ISM.

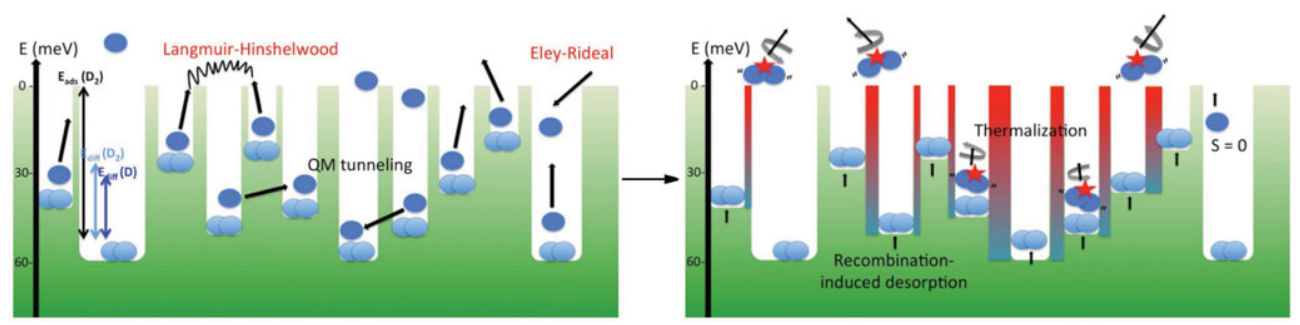

Figure 1. Enhanced formation due to pre-existing molecules on a silicate at $\mathrm{T}=10 \mathrm{~K}$.

$\dagger$ Current adress: Physics Department, Syracuse University, Syracuse, NY 13244, USA. 